Ferrata Storti Foundation

\title{
Oncogenic fusion protein BCR-FGFR1 requires the breakpoint cluster region-mediated oligomerization and chaperonin Hsp90 for activation
}

Haematologica 2020

Volume 105(5):1262-1273

\section{Correspondence:}

DANIEL J. DONOGHUE

ddonoghue@ucsd.edu

Received: March 1, 2019.

Accepted: August 14, 2019.

Pre-published: August 22, 2019.

doi:10.3324/haematol.2019.220871

Check the online version for the most updated information on this article, online supplements, and information on authorship \& disclosures: www.haematologica.org/content/105/5/1262

(C)2020 Ferrata Storti Foundation

Material published in Haematologica is covered by copyright. All rights are reserved to the Ferrata Storti Foundation. Use of published material is allowed under the following terms and conditions:

https://creativecommons.org/licenses/by-nc/4.0/legalcode. Copies of published material are allowed for personal or internal use. Sharing published material for non-commercial purposes is subject to the following conditions:

https://creativecommons.org/licenses/by-nc/4.0/legalcode, sect. 3. Reproducing and sharing published material for commercial purposes is not allowed without permission in writing from the publisher.

\author{
Malalage N. Peiris, ${ }^{1}$ April N. Meyer, ${ }^{1}$ Katelyn N. Nelson, ${ }^{1}$ Ezra W. Bisom-Rapp, ${ }^{1}$ \\ and Daniel J. Donoghue ${ }^{1,2}$
}

${ }^{1}$ Department of Chemistry and Biochemistry, University of California San Diego and ${ }^{2}$ Moores Cancer Center, University of California San Diego, La Jolla, CA, USA

\section{ABSTRACT}

M utation and translocation of fibroblast growth factor receptors often lead to aberrant signaling and cancer. This work focuses on the $t(8 ; 22)(p 11 ; q 11)$ chromosomal translocation which creates the breakpoint cluster region (BCR) fibroblast growth factor receptor1 (FGFR1) (BCR-FGFR1) fusion protein. This fusion occurs in stem cell leukemia/lymphoma, which can progress to atypical chronic myeloid leukemia, acute myeloid leukemia, or B-cell lymphoma. This work focuses on the biochemical characterization of BCR-FGFR1 and identification of novel therapeutic targets. The tyrosine kinase activity of FGFR1 is required for biological activity as shown using transformation assays, interleukin-3 independent cell proliferation, and liquid chromatography/mass spectroscopy analyses. Furthermore, BCR contributes a coiled-coil oligomerization domain, also essential for oncogenic transformation by BCR-FGFR1. The importance of salt bridge formation within the coiled-coil domain is demonstrated, as disruption of three salt bridges abrogates cellular transforming ability. Lastly, BCR-FGFR1 acts as a client of the chaperonin heat shock protein 90 (Hsp90), suggesting that BCR-FGFR1 relies on Hsp90 complex to evade proteasomal degradation. Transformed cells expressing BCR-FGFR1 are sensitive to the Hsp90 inhibitor Ganetespib, and also respond to combined treatment with Ganetespib plus the FGFR inhibitor BGJ398. Collectively, these data suggest novel therapeutic approaches for future stem cell leukemia/lymphoma treatment: inhibition of BCR oligomerization by disruption of required salt bridges; and inhibition of the chaperonin Hsp90 complex.

\section{Introduction}

Fibroblast growth factor receptors (FGFR) are part of the receptor tyrosine kinase (RTK) family and are responsible for cell growth and proliferation. The FGFR family is composed of four homologous receptors; all contain three extracellular immunoglobulin-like domains, a transmembrane domain, and a split kinase domain. When these receptors are bound to fibroblast growth factor (FGF) and heparin sulfate proteoglycans, they are able to dimerize, which leads to auto-phosphorylation of the kinase domain and activation of downstream cell signaling pathways including signal transducer and activator of transcription (STAT), mitogen activated protein kinase (MAPK), protein kinase B (AKT), and phospholipase C gamma (PLC $\gamma$ ). FGFR signaling results in cellular migration, cell proliferation, angiogenesis, and wound healing. ${ }^{1}$

FGFRs are often aberrantly activated in cancer by overexpression, mutation, or translocation. Specifically, FGFR1 is involved in stem cell leukemia/lymphoma (SCLL), also known as 8p11 myeloproliferative syndrome (EMS). ${ }^{2}$ SCLL is characterized by a chromosomal translocation that produces a dimerizing protein partner fused to the kinase domain of FGFR1. ${ }^{3}$ Although SCLL is rare, it can aggressively progress to atypical chronic myeloid leukemia (CML), acute myeloid leukemia (AML), or B-cell lymphoma. Despite extensive chemotherapy, the only known cur- 
ative option for SCLL patients is hematopoietic stem cell transplantation. Although both Ponatinib and Pemigatinib (INCB054828) have been used to treat patients with mixed results, few other alternative treatment plans exist for patients who are either awaiting or are unable to receive transplantation. ${ }^{4,5}$ This work focuses on the $\mathrm{t}(8 ; 22)(\mathrm{p} 11 ; \mathrm{q} 11)$ chromosomal translocation resulting in the BCR-FGFR1 fusion protein with exon 4 of the breakpoint cluster region (BCR) fused to exon 9 of FGFR1. Although BCR was first identified fused to Abelson murine leukemia viral oncogene homolog-1 (ABL), also known as the Philadelphia chromosome, BCR has since then been identified fused to ret proto-oncognene (RET), Janus kinase 2 (JAK2), and platelet derived growth factor receptor alpha (PDGFRA) ${ }^{6-9}$ Although a common fusion partner, the endogenous function of the $B C R$ gene remains obscure. The fusion protein BCR-FGFR1 retains the coiled-coil dimerization/oligomerization domain, putative serine/threonine kinase domain, and partial RhoGEF domain from BCR. ${ }^{10}$

The BCR-FGFR1 fusion is not well characterized, and this work seeks to elucidate the underlying mechanisms behind BCR-FGFR1 mediated SCLL. Although tyrosine kinase inhibitor therapies (TKI) are traditionally used to treat certain hematological cancers, the use of TKI often results in drug resistance in patients. Thus, it is crucial to determine additional therapeutic strategies in treating hematopoietic cancers. Here we suggest disruption of the BCR coiled-coil dimerization domain and Hsp90 inhibition as novel therapeutic targets for BCR-FGFR1 driven SCLL. Data presented here may also allow for additional approaches in treating BCR-ABL mediated CML, due to the similarity between BCR-ABL and BCR-FGFR1 fusion proteins.

\section{Methods}

\section{DNA Constructs}

The $B C R$ gene (pSG65-Bcr) was purchased from Addgene (Watertown, MA, USA) and was subcloned into pcDNA3. FGFR1 and FGFR1-K656E were previously described. ${ }^{11}$ To construct $B C R$ FGFR1, a BamHI site was introduced by PCR-based site-directed mutagenesis after amino acid L584 in BCR and before amino acid V429 in FGFR1. This unique internal BamHI site was used together with an upstream site of EcoRI to subclone the 5' region of $B C R$ into the FGFR1 pCDNA3 plasmid, creating a fusion breakpoint of BCR exon 4 fused to FGFR1 exon 9. The BamHI site adds 6 bases which code for a GS linker between the 5' BCR and the 3' FGFR1. FGFR1-K514A $A^{12}$ and all other mutations described were introduced by PCR-based site-directed mutagenesis. FGFR1 or BCRFGFR1 clones were subcloned into the pLXSN expression plasmid for use in NIH3T3 or 32D cells. Details of plasmid DNA used are in the Online Supplementary Material and Methods.

\section{Cell transfection, immunoprecipitation, immunoblot analysis}

HEK293T cells were transfected with $3 \mu \mathrm{g}$ of the pcDNA3 plasmid constructs using calcium phosphate transfection as described. ${ }^{13}$ Immunoblotting was performed as described. ${ }^{14}$ NIH3T3 focus assays were performed as described. ${ }^{15}$ Number of foci were, normalized by transfection efficiency, and quantitated relative to a positive control +/-standard error of the mean (SEM).

The Hsp90 inhibitor, Ganetespib was added to HEK293T transfected cells at a concentration of $200 \mathrm{nM}$ for 4 hours (h) during cell starvation at $10 \% \mathrm{CO} 2,37^{\circ} \mathrm{C}$. Transfected NIH3T3 cells were maintained with $0,10,20,23,25$, or $30 \mathrm{nM}$ Ganetespib for 14 days, and fixed and scored as described. For combination drug treatment, $15 \mathrm{nM}$ Ganetespib was used with the FGFR inhibitor BGJ398 at $0,2.5$, or $10 \mathrm{nM}$. All cell assays were performed a minimum of three times. More detailed information is provided in the Online Supplementary Materials and Methods.

\section{Interleukin-3 (IL-3) independent growth in 32D cells}

A total of $1 \times 10^{6} 32 \mathrm{D}$ cells were electroporated $(1,500 \mathrm{~V}, 10 \mathrm{~ms}, 3$ pulses) by Neon Transfection system using $30 \mu \mathrm{g}$ of FGFR 1 or $B C R$-FGFR 1 derivatives in pLXSN in triplicate. $48 \mathrm{~h}$ after transfection, cells were selected with $1.5 \mathrm{mg} / \mathrm{mL}$ Geneticin (G418) for 10 days to generate stable cell lines before starting IL- 3 independent growth assays. Triplicate flasks were seeded with the cell lines at $4 \times 10^{4}$ cell $/ \mathrm{mL}$ in the presence or absence of mouse IL-3. In addition, $1 \mathrm{nmol} / \mathrm{L}$ of FGF and $30 \mathrm{ug} / \mathrm{mL}$ of heparin was added to a set of flasks in the absence of IL-3. On days 1, 3, 5, 7, and 9 samples were counted and measured for MTT metabolic activity as described. ${ }^{16}$ For Ganetespib treatment, cells were seeded with 0 , 2.5, or 5.0 nM Ganetespib -/+ IL-3. MTT metabolic activity was measured on days 3,5 , and 7 . A concentration of $10 \mathrm{nM}$ or higher of Ganetespib was found to be toxic to 32D cells in the presence of IL-3.

\section{Mass spectrometry sample preparation}

Liquid chromatography/mass spectroscopy (LC-MS/MS) for phosphopeptide analysis was as described previously. ${ }^{16}$ Complete information is available in the Online Supplementary Material and Methods.

\section{Results}

\section{Signaling cascade activation by BCR-FGFR1}

The role of BCR in this fusion protein has remained unclear, as BCR-FGFR1 retains the coiled-coil oligomerization domain, putative serine/threonine kinase domain, and partial RhoGEF domain from BCR. In order to elucidate if BCR-FGFR1 relies on the tyrosine kinase activity of FGFR1, a K514A kinase dead mutation, ${ }^{12}$ or a K656E kinase activating mutation, ${ }^{11,15}$ were introduced in the FGFR1 tyrosine kinase domain in both FGFR1 and BCRFGFR1 backgrounds (Figure 1A). These studies were performed in HEK293T cells, as they have previously been used in FGFR signal transduction and protein phosphorylation studies. ${ }^{16}$ HEK293T cells expressing either fulllength FGFR1 or BCR-FGFR1 variants were analyzed for MAPK, STAT3 and STAT5 activation, and FGFR1 receptor phosphorylation. Expression of FGFR1 shows slight activation of the MAPK pathway, while expression of the activated FGFR1-K656E shows elevated phosphorylation levels of MAPK (Figure 1B, panel 7). Expression of BCRFGFR1 or BCR-FGFR1-K656E also resulted in MAPK phosphorylation. Additionally, STAT3 and STAT5 phosphorylation were strongly elevated by BCR-FGFR1 and BCRFGFR1-K656E, in comparison to FGFR1 or FGFR1-K656E, indicating that BCR-FGFR1 induces both MAPK and STAT pathway activation (Figure 1B, panel 3 and 5).

To examine the phosphorylation of each fusion construct compared with FGFR1, FGFR1-K656E and FGFR1K514A were expressed in HEK293T cells, collected and immunoprecipitated with a C-terminal FGFR1 antibody, and probed for tyrosine phosphorylation. An increase in tyrosine phosphorylation was observed in both BCR- 


\section{A FGFR1}

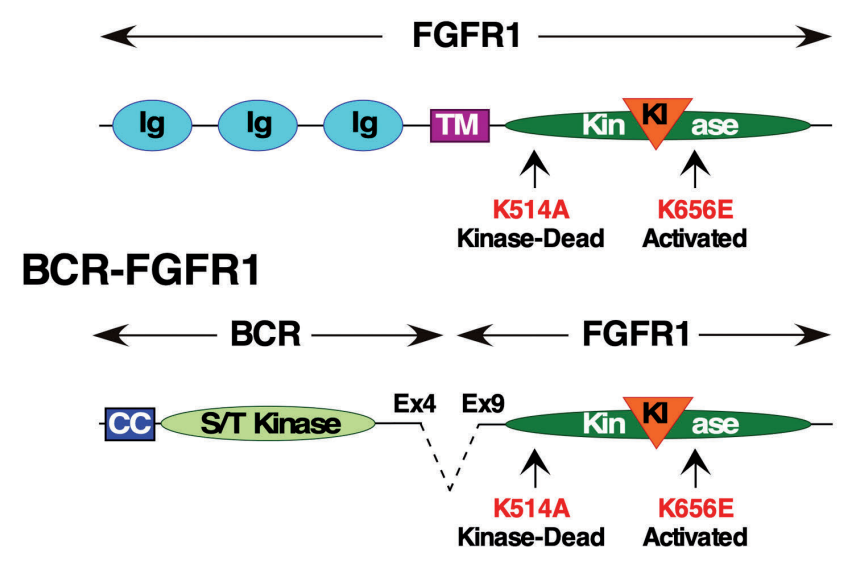

B
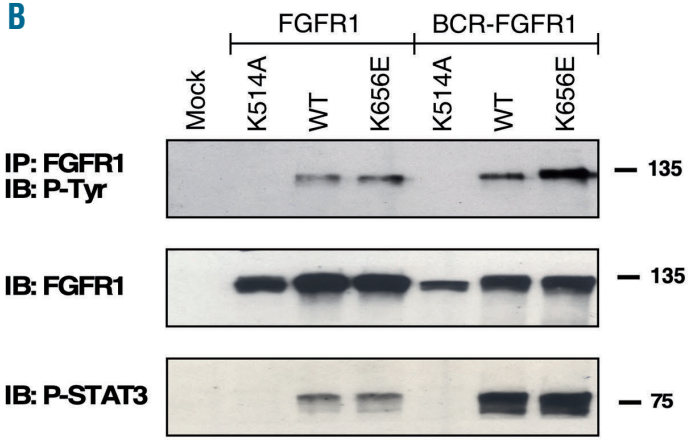

IB: STAT3
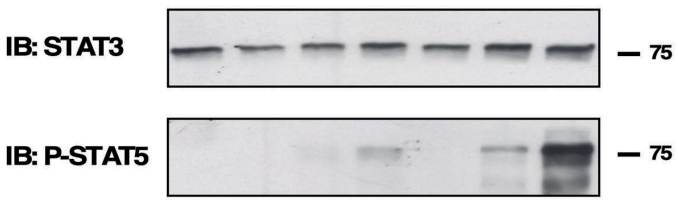

IB: STAT5

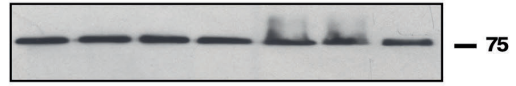

IB: P-MAPK

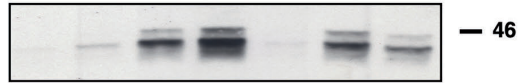

IB: MAPK

Figure 1. Cell signaling activated by BCR-FGFR1. (A) Schematic of FGFR1 and BCR-FGFR1 with K514A kinase dead, and K656E kinase activating mutations shown. FGFR1 contains an extracellular ligand binding domain with immunoglobulin-like domains (Ig), a transmembrane domain (TM), a split tyrosine kinase domain, and kinase insert domain (KI). BCR-FGFR1 contains breakpoint cluster region (BCR) exon 4 at the N-terminus fused to the kinase domain of fibroblast growth factor receptor 1 (FGFR1) at exon 9. BCR contributes a coiled-coil (CC) and a putative serine/threonine (S/T) kinase domain to the BCR-FGFR1 fusion. (B) Lysates of HEK293T cells expressing either FGFR1 or BCR-FGFR1 derivatives were immunoprecipitated with anti-FGFR1 antibody and immunobotted with phosphotyrosine antibody (panel 1). These lysates were immunoblotted with anti-FGFR1 antibody to detect expression of transfected clones (panel 2). Lysates were examined for activation of the STAT3, STAT5 and MAPK pathways using phospho-specific antibodies; phospho-STAT3 (Y705) (panel 3), phospho-STAT5 (Y694) (panel 5) and phospho-MAPK (T202/Y204) (panel 7). Membranes were stripped and reprobed for total STAT3, STAT5 and MAPK shown in panels 4, 6 and 8, respectively.

FGFR1, and BCR-FGFR1-K656E expressing cells, when compared to FGFR1, indicating that the contribution of $B C R$ as a partner gene to this fusion increases the constitutive phosphorylation of FGFR1 (Figure 1B, top panel). Interestingly, although the putative serine/threonine kinase domain in BCR is present in the BCR-FGFR1 fusion protein, HEK293T cells expressing BCR-FGFR1-K514A, which contains BCR fused to a kinase-dead FGFR1, does not activate MAPK or STAT pathways (Figure 1B). In addition, no tyrosine phosphorylation of FGFR1 was detected for cells expressing BCR-FGFR1-K514A, suggesting that BCR-FGFR1 relies on the constitutive kinase activity of FGFR1 for activation of downstream cell signaling.

Cell transforming ability of BCR-FGFR1 by focus assay

In order to investigate the transforming ability of BCRFGFR1 and subsequent mutants, these constructs were assayed in a NIH3T3 cell transforming assay. NIH3T3 cell transformation assays were one of the original assays used to discover and characterize novel oncogenes such as activated RAS, MUC4, AKT and various other oncogenes. ${ }^{16,17}$ NIH3T3 cells expressing BCR-FGFR1, BCR-FGFR1-K656E, and FGFR1-K656E exhibited high levels of focus formation (Figure 2B, D and G). FGFR3-TACC3, a known fusion oncogene, ${ }^{15,16}$ was used as a positive control. Interestingly, BCR-FGFR1 and BCR-FGFR1-K656E exhibited nearly three-fold higher focus formation in comparison to FGFR3-TACC3. NIH3T3 cells expressing either FGFR1, FGFR1-K514A, or BCR-FGFR1-K514A did not form any visible foci (Figure 2C, E-F), indicating that the kinase activity of FGFR1 is critical to the transforming ability of this fusion.

\section{BCR-FGFR1 promotes IL-3 independent cell growth}

The BCR-FGFR1 fusion protein has been solely discovered in hematopoietic cancers to date. Previous studies have utilized either $\mathrm{Ba} / \mathrm{F} 3$ or 32D hematopoietic cell lines to demonstrate oncogenic and proliferative potential in these IL-3 dependent cell lines. ${ }^{16,18} 32 \mathrm{D}$ cells were used to investigate the proliferative potential of cells expressing FGFR and BCR-FGFR1 derivatives in the presence and absence of IL-3, and in the absence or presence of aFGF (Figure 3).

FGFR1, FGFR1-K656E, BCR-FGFR1, and BCR-FGFR1$K 656$ E were electroporated into $32 \mathrm{D}$ cells. Only 32D cells which expressed either BCR-FGFR1 or BCR-FGFR1K656E were able to proliferate in the absence of IL-3, as seen through MTT metabolic assays and cell counts (Figure $3 \mathrm{~A}$ and $\mathrm{D}$ ). The addition of aFGF in the absence of IL-3 resulted in a slight increase in cell growth for FGFR1 and FGFR1-K656E, but no change for BCR-FGFR or BCRFGFR1-K656E (Figure 3B and E), as expected since the 


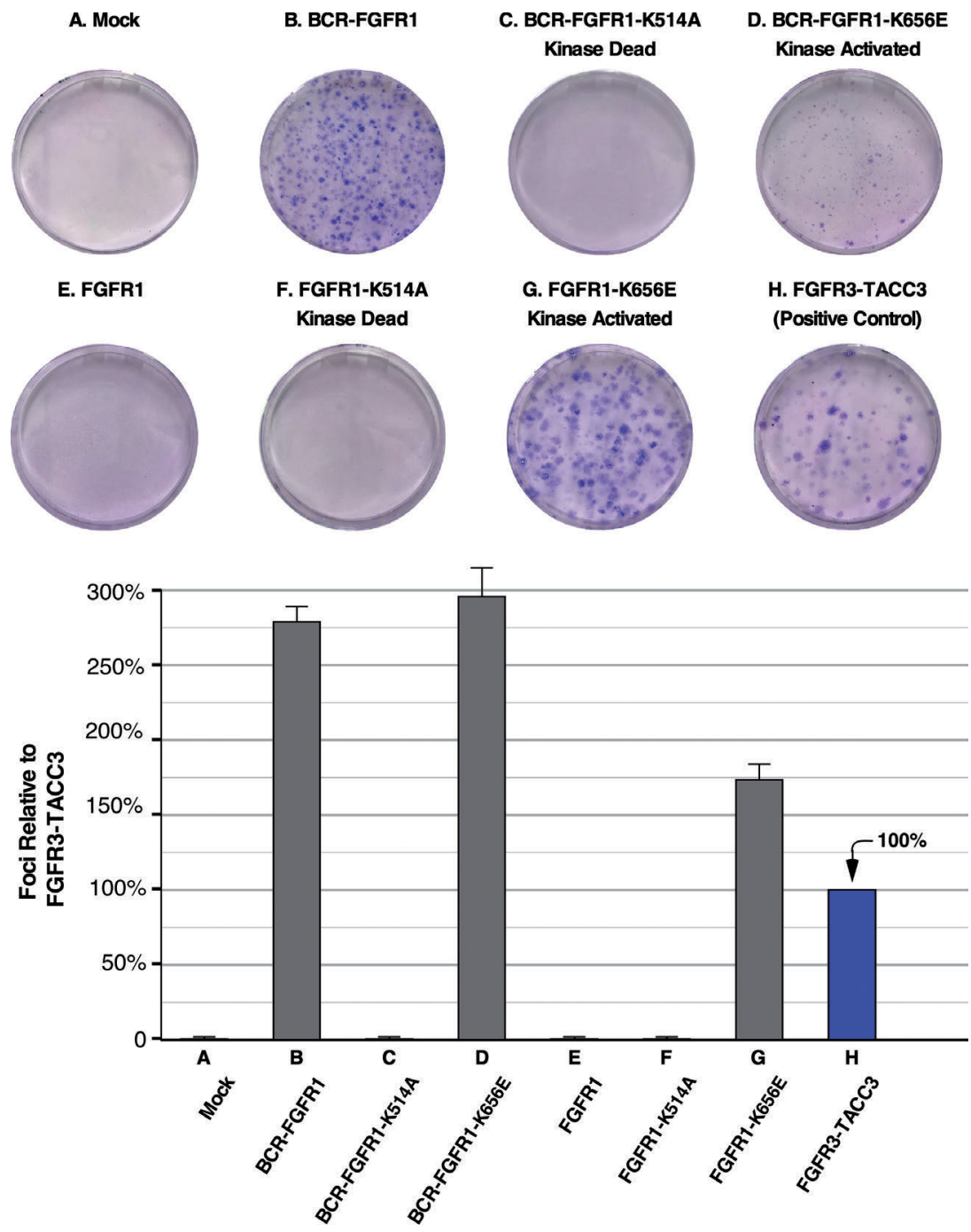

Figure 2. Cell transformation of NIH3T3 cells by BCR-FGFR1. Representative plates from a focus assay are shown, with transfected constructs indicated. The graph shows the number of foci scored, normalized for transfection efficiency and calculated as a percentage of transformation relative to FGFR3-TACC3 -/+ standard error of the mean (SEM). Assays were performed a minimum of three times per each DNA construct.

extracellular ligand binding domain of FGFR1 is removed in the fusion proteins. All transfected constructs displayed cell viability in the presence of IL-3 (Figure 3C and F). Thus, these data demonstrate that the BCR-FGFR1 fusion protein requires the $\mathrm{N}$-terminal contribution of $\mathrm{BCR}$ for IL-3-independent proliferation as assayed in 32D cells.

\section{LC-MS/MS analysis identifies novel phosphorylation} sites

The strong tyrosine phosphorylation signal seen in BCR-FGFR1 and BCR-FGFR1-K656E lysates though immunoblotting (Figure 1) led to the inquiry of whether there were any novel phosphorylation sites within these fusion proteins. To investigate this question, HEK293T cell lysate expressing either FGFR1 or BCR-FGFR1 derivatives were immunoprecipitated and analyzed by LCMS/MS as described. ${ }^{16}$

Both BCR-FGFR1 and BCR-FGFR1-K656E exhibit high phosphorylation levels on key tyrosine residues in the FGFR1 kinase domain, whereas BCR-FGFR1-K514A exhibited only slight phosphorylation (Figure 4). The lack of phosphorylation on the activation loop tyrosine residues in BCR-FGFR1-K514A indicates that FGFR1 kinase activity is critical for activation of the BCR-FGFR1 fusion.
Additional serine, threonine, and tyrosine phosphorylation sites were also detected in BCR-FGFR1 or BCRFGFR1-K656E (Figure 4B-C) which have not been previously reported. To determine the role of these novel phosphoacceptor sites within the BCR domain of BCR-FGFR1 fusion proteins, phosphorylated serine or threonine residues were mutated to alanine, and phosphorylated tyrosine residues in $B C R$ were mutated to phenylalanine. Each construct was assayed for focus formation. In addition to these mutations, a BCR(Y177F)-FGFR1 mutant was also assayed as it mutates the Grb2 binding site, previously shown to reduce activation of the BCR-FGFR1 fusion protein. ${ }^{19,20}$

The Y177F Grb2 mutation in BCR-FGFR1 shows a 50\% decrease in transforming ability when compared to NIH3T3 cells expressing BCR-FGFR1. However, all additional mutations in BCR phosphoacceptor sites displayed little to no difference in transforming ability (Table 1). The mutant BCR(T359A/S367A/S369A/S377A)-FGFR1, which mutates phosphorylated residues within the $\mathrm{ABL} \mathrm{SH} 2$ binding domain present in BCR, was also transforming. Taken together, LC-MS/MS data and cell transformation assays suggest that tyrosine phosphorylation on activation loop residues within the FGFR1 kinase domain is critical for BCR-FGFR1 activation. BCR-FGFR1 relies on an active 
A

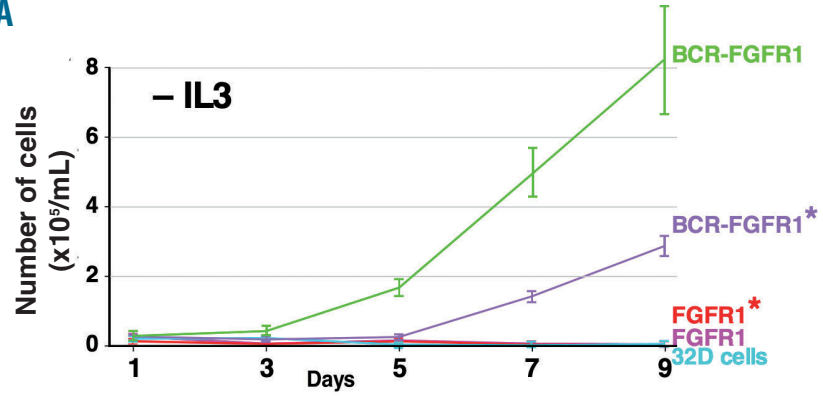

B

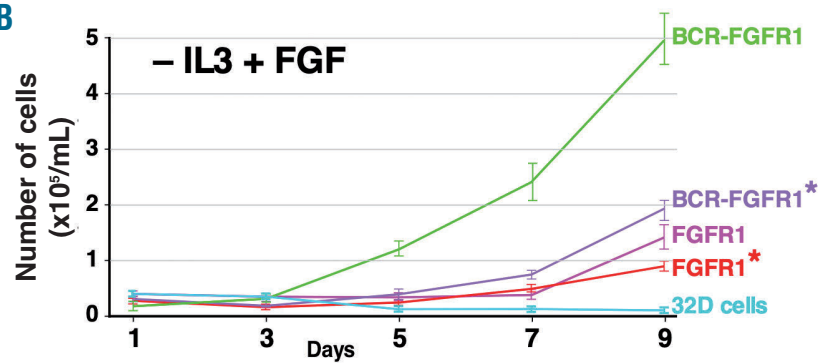

C

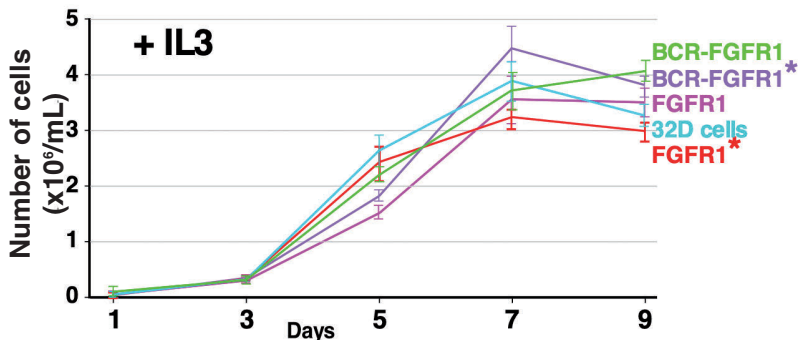

D
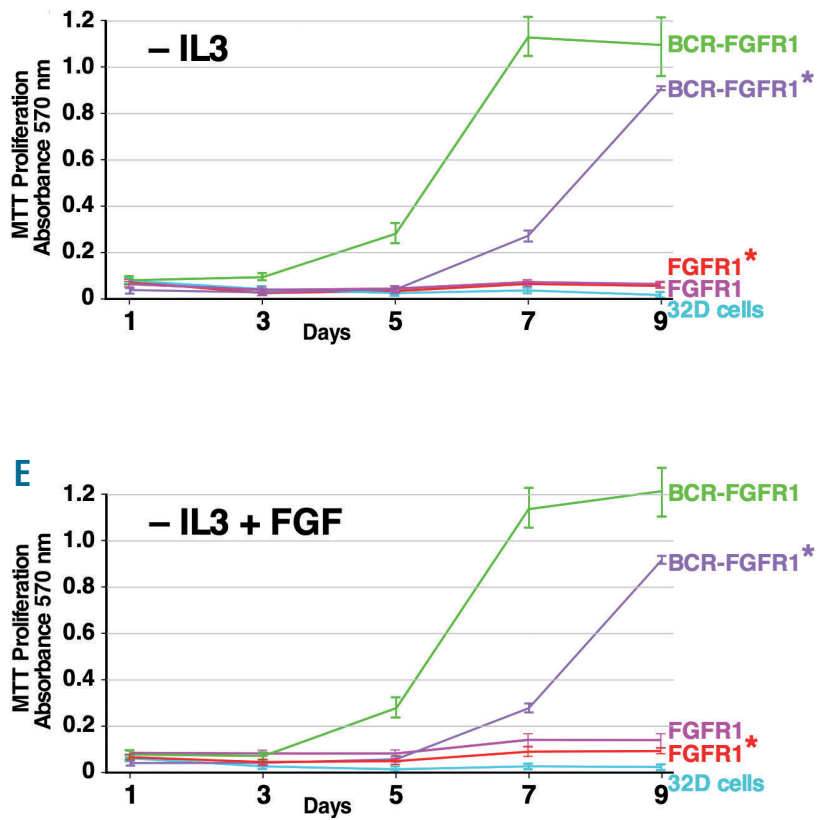

F

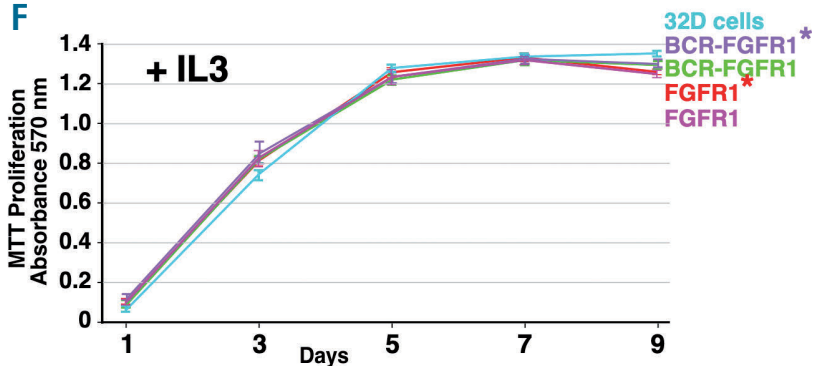

Figure 3. BCR-FGFR1 supports interleukin-3-independent proliferation. 32D control cells and cell lines selectively expressing FGFR1, FGFR1-K656E (FGFR1*), BCRFGFR1 and BCR-FGFR1-K656E (BCR-FGFR1*) were cultured in the absence of interleukin-3 (IL-3) (A and D), in the presence of aFGF/Heparin (B and E) and in the presence of IL-3 (C and F). Total number of cells were counted on days 1, 3, 5, 7 and 9 to examine IL-3 independent growth (A, B, and C). Cell viability was determined by MTT metabolic assay (D, E, and F). All control cells and cell lines were assayed in triplicate. Standard deviation is shown.

FGFR1 kinase domain for transformation, while phosphorylation on residues within the BCR domain does not appear to be critical.

\section{Salt bridge disruption in BCR dimerization domain abrogates cell transforming ability}

The coiled-coil oligomerization domain of BCR is an attractive therapeutic target, as it is essential for cell transformation as demonstrated with assays done in BCRABL. ${ }^{21}$ Previous work has shown the necessity of salt bridge formation for the activation of oncogenic fusion protein ETV6-NTRK3, which is found in AML. ${ }^{22}$ Here we investigated the importance of salt bridge formation in the BCR coiled-coil domain as a potential requirement for the oligomerization and oncogenic activation of BCR-FGFR1.

The coiled-coil oligomerization region of BCR spans amino acid residues 3-75 and has been proposed to contain two interhelical salt bridges stabilizing the two coils in an antiparallel coiled-coill ${ }^{23}$ (Figure 5). The first salt bridge (Salt Bridge \#1) was proposed to form between residues Glu34 and Arg55, whereas the second salt bridge (Salt Bridge \#2), between Glu46 and Arg53 is visible in the crystal structure of the BCR oligomerization domain (Figure $5 \mathrm{~B}){ }^{23-25}$ Each salt bridge residue was individually mutated to a residue of the opposite charge to abolish the electrostatic interaction. When residues Glu34 or Arg55 in BCR Salt Bridge \#1 were mutated to the opposing charge, E34R or R55E respectively, little to no effect was observed in cell transformation (Figure 5C, mutants 2 and 8). Likewise, when residues Glu46 or Arg53 in BCR Salt Bridge \#2 were mutated to the opposing charge, E46R or R53E respectively, no effect in cell transformation was observed (Figure 5C, mutants 3-4).

We also became aware of the possible importance of a putative intrahelical salt bridge (Salt Bridge \#3) involving E52 and R55 $5^{23}$ (Figure 5A-B). Since R55 is also involved in Salt Bridge \#1, this means that mutation of R55E to probe the importance of Salt Bridge \#1 inadvertently disrupts the intrahelical Salt Bridge \#3. This extra layer of complexity was analyzed as shown in Table 2. The upper portion of 


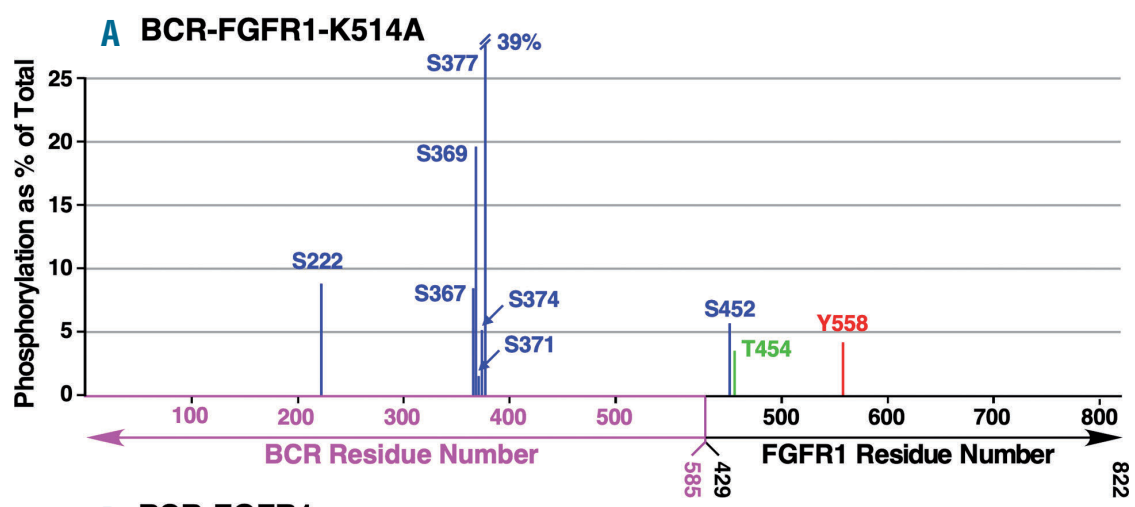

Figure 4. Phosphorylated residues in BCR-FGFR1 derivatives identified by mass spectrometry analysis. FGFR1 and BCR-FGFR1 derivatives were transfected into HEK293T cells and examined fo phosphorylation by mass spectrometry as described in the Methods. The phosphorylation sites are indicated that were greater than $1 \%$ of the total phosphorylation detected in the sample. P-Tyr is shown in red; P-Thr is shown in green; P-Ser is shown in blue. Duplicate, independent samples were averaged; (A) BCR FGFR1-K514A, (B) BCR-FGFR1, (C) BCR-FGFR1-K656E. There was no significant difference in the phosphorylation sites detected in the FGFR1 kinase domains in BCR FGFR1 fusions compared to the FGFR1 derivatives (data not shown). (D) A schematic presentation of the BCR-FGFR1 fusion protein with key amino acid positions and domains labeled. The oligomerization domain (OLIGO), the Grb2 and the Abl SH2 binding domains are shown. Also shown is the partial DBL-homologous (DBL) domain, which is a structural RhoGEF domain of guanine nucleotide exchange factors for Rho/Rac/Cdc42-like GTPases.

C BCR-FGFR1-K656E
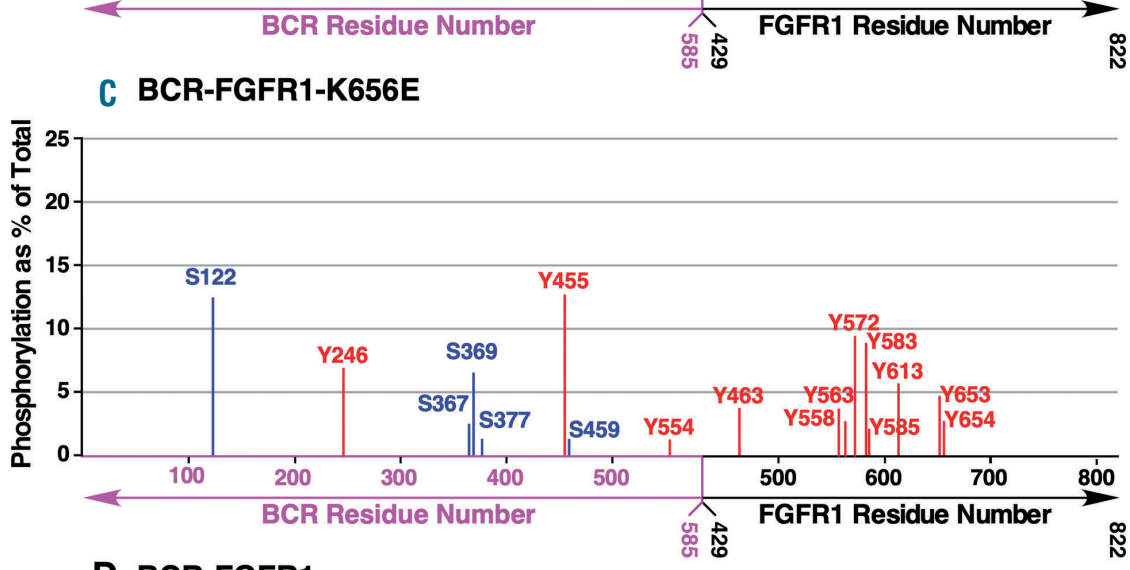

\section{BCR-FGFR1}

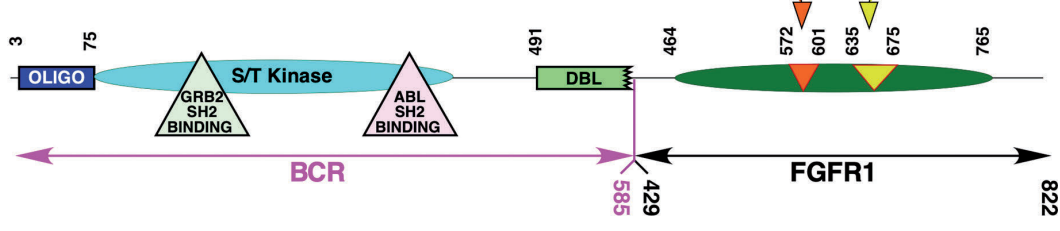

the table lists all possible mutations in Salt Bridges \#1 and \#2 (mutants 2-5) which do not disrupt Salt Bridge \#3. The lower portion of the table lists all possible mutations in Salt Bridges \#1 and \#2 which also perturb Salt Bridge \#3 (mutants 7-12).

When only Salt Bridge \#3 was disrupted in the E52R mutation (Figure 5C, mutant 6), little effect was observed. When various combinations of mutations were examined, affecting Salt Bridge \#3 together with either Salt Bridge \#1 or Salt Bridge \#2, the effects on biological activity were variable (Figure 5C, mutants 7-10). However, cell-transforming ability by BCR-FGFR1 was significantly reduced or completely abrogated when all three salt bridges were disrupted simultaneously: the mutant E34R/E46R/E52R exhibited $<1 \%$ transformation activity, and the mutant R53E/R55E exhibited only 14\% (Figure 5C, mutants
11-12). These data suggest that these three salt bridges in the BCR coiled-coil domain together provide a critical and partially redundant role in the oligomerization and activation of BCR-FGFR1. Figure 5D presents focus assay plates for BCR-FGFR1 (Figure 5D, mutant 1), and for selected mutants that disrupt either 2 or 3 salt bridge simultaneously (Figure 5D, mutants 5, 7, 9-12).

\section{BCR-FGFR1 is an HSP90 addicted oncoprotein}

Hsp90 is a highly conserved, ubiquitously expressed molecular chaperone that controls the stability of certain proteins. ${ }^{26}$ Prior work has shown that Hsp90 is overexpressed in certain cancers, and the Hsp90 complex provides stability for various oncogenic proteins, which are necessary for cancer cell survival. ${ }^{27}$ Many of these oncogenes, such as mutated P53 or BCR-ABL take advantage of 
the Hsp90 chaperone system to avoid ubiquitination and proteasomal degradation. ${ }^{28}$ Here, we aim to uncover if BCR-FGFR1 is a client of Hsp90 and possibly relies on the Hsp90 complex for stability and cellular survival.

HEK293T cell lysate expressing either FGFR1 or BCRFGFR1 derivatives were immunoprecipitated with FGFR1 antisera and immunoblotted for Hsp90. An interaction was observed between Hsp90 and BCR-FGFR1 derivatives (Figure 6A). To further analyze if BCR-FGFR1 is dependent on Hsp90 for cellular stability and activity, assays with potent Hsp90 inhibitor, Ganetespib, were performed. HEK293T cells expressing either FGFR1 or BCR-FGFR1 derivatives were treated with $200 \mathrm{nM}$ Ganetespib for $4 \mathrm{~h}$, then analyzed for overall FGFR1 expression and activation of downstream cell signaling pathways (Figure 6B). A significant reduction in BCRFGFR1 expression is observed following Ganetespib
Table 1. Biological activity of mutations in phosphoacceptor sites.

\begin{tabular}{lcc} 
Construct & $\begin{array}{c}\text { Foci relative to } \\
\text { BCR-FGF1 (\%) }\end{array}$ & SEM (\%) \\
Mock & 0 & 0 \\
BCR-FGFR1 & 100 & 11 \\
\hline BCR(Y177F)-FGFR1 & 55 & 16 \\
BCR(Y436F)-FGFR1 & 120 & 2 \\
\hline BCR(Y455F)-FGFR1 & 108 & 9 \\
BCR(S122A)-FGFR1 & 97 & 1 \\
\hline BCR(Y246F)-FGFR1 & 105 & 7 \\
BCR(S459A)-FGFR1 & 129 & 1 \\
\hline BCR(Y554F)-FGFR1 & 126 & 11 \\
BCR(T359A/S367A/S369A/S377A)-FGFR1 & 129 & 3
\end{tabular}

BCR: breakpoint cluster region; FGFR1: fibroblast growth factor receptor 1; SEM: standard error of the mean.

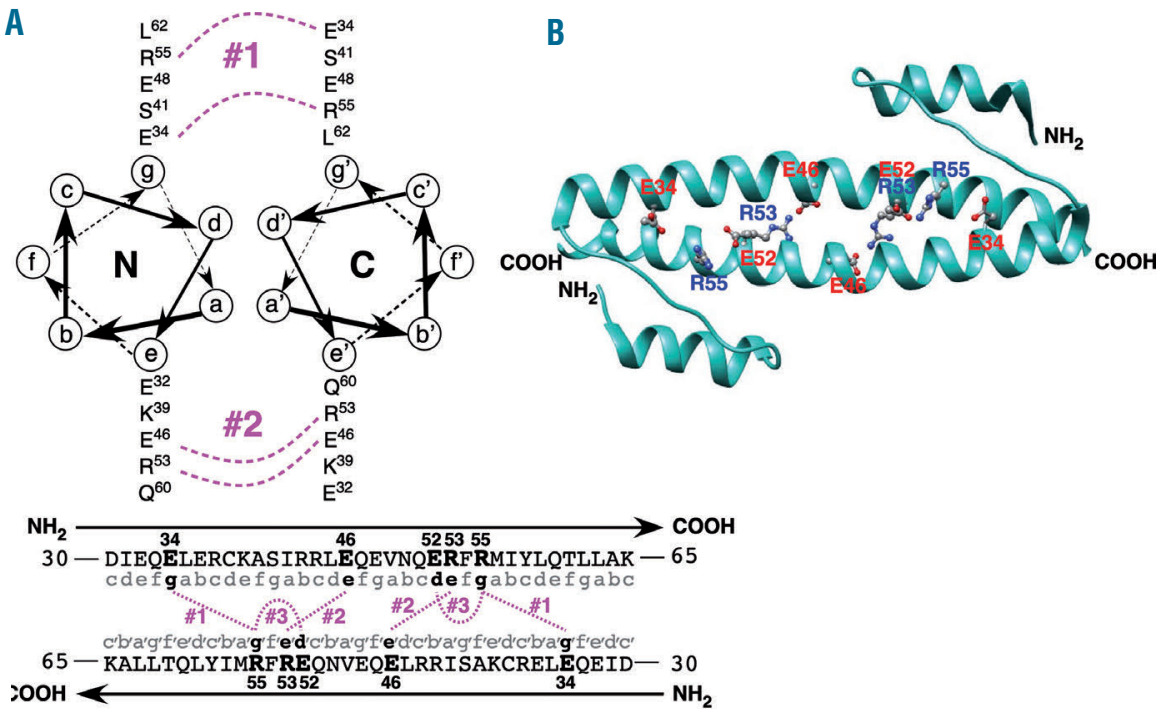

C

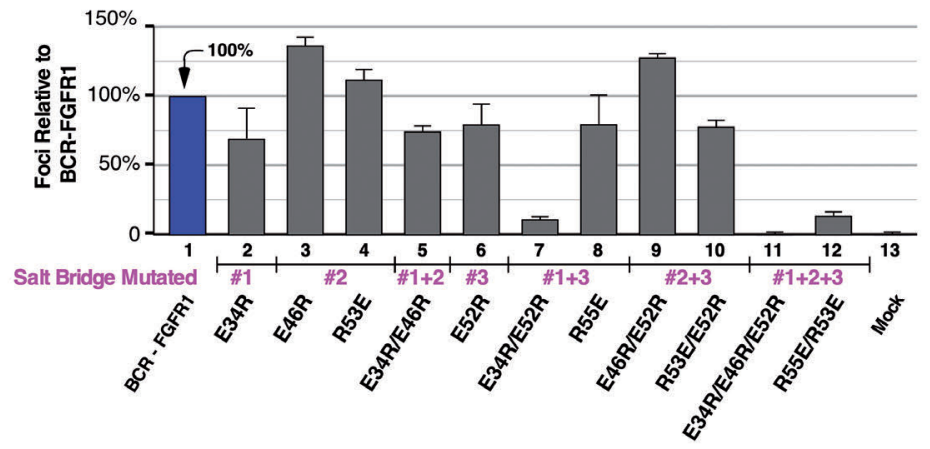

D

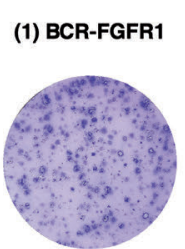

(10) R53E / E52R

Salt bridge \#2+3

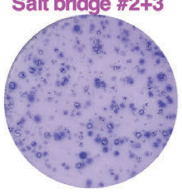

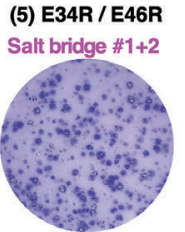

(11) E34R / E46R / E52R Salt bridge \#1+2+3

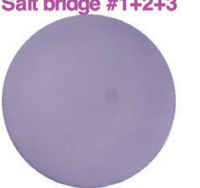

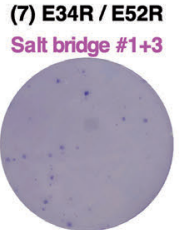

(12) R55E / R53E Salt bridge \#1+2+3

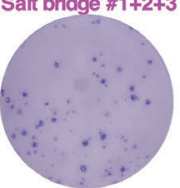

(9) E46R / E52R

Salt bridge \#2+3

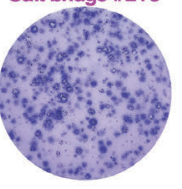

(13) Mock

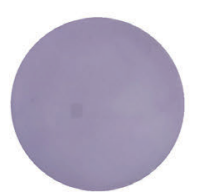

Figure 5. Three salt bridges are required for BCRFGFR1 activity. Focus assay results with salt bridge mutations made in BCR coiled-coil domain (A) The anti-parallel heptad repeats of the coiledcoil domain of BCR showing the residues in the "e" and "g" positions. ${ }^{23}$ The two interhelical salt bridges are indicated between residues E34 and R55 (\#1) in the "g" positions, and between E46 and R53 (\#2) in the "e" positions. Below, residues 30 through 65 of the anti-parallel region are shown with the salt bridges indicated. Also shown in the linear sequence is the location of the intrahelical salt bridge involving E52-R55 in the " $\mathrm{d}$ " and "g" positions. (B) The crystal structure of the breakpoint cluster region (BCR) coiled-coil oligomerization domain is shown (PDB code $1 \mathrm{~K} 1 \mathrm{~F}$ ), as viewed using Chimera software. ${ }^{24,25}$ Positive residues R53 and R55 are in blue while negative residues E34, E46, and E52 are in red. (C) Results from NIH3T3 transformation assay expressing BCR-FGFR1 fusions with salt bridge mutations. Foci were scored, normalized for transfection efficiency and quantitated relative to BCRFGFR1 -/+ standard error of the mean (SEM). Each DNA construct was assayed at least three times. (D) Representative plates from a focus assay are shown. In the interest of space, plates are shown only for selected mutants that disrupt either two or three salt bridges simultaneously, as indicated. 
treatment (Figure 6B, top panel). Furthermore, treatment with this Hsp90 inhibitor shows a decrease in phosphorylated STAT3, and complete ablation of phosphorylated Tyr on BCR-FGFR1, when compared to control cells treated (Figure 6B, panel 4 and 6). Interestingly, MAPK retains phosphorylation, despite the loss of phosphorylated BCR-FGFR1 (Figure 6B, panel 2). Although unexpected, this result could be due to the Grb2 binding site present in BCR, which activates downstream Ras and MAPK pathways independently of FGFR1 activation. ${ }^{19}$ The dra-

Table 2. Mutations affecting interhelical Salt Bridges \#1 and/or \#2, and intrahelical Salt Bridge \#3. Mutated residues shown in bold font; missing salt bridge indicated by $\otimes$. Wt: wild-type.

\begin{tabular}{|c|c|c|c|c|c|}
\hline Mutant Name & $\begin{array}{l}\text { Mutated Salt } \\
\text { Bridges }\end{array}$ & $\begin{array}{c}\text { Salt Bridge \#1 } \\
\text { Residues }\end{array}$ & $\begin{array}{c}\text { Salt Bridge \#2 } \\
\text { Residues }\end{array}$ & $\begin{array}{c}\text { Salt Bridge \#3 } \\
\text { Residues }\end{array}$ & Activity \\
\hline (1) BCR-FGFR1 WT & None & $\mathrm{E} 34 \bullet \mathrm{R} 55$ & $\mathrm{E} 46 \bullet \mathrm{R} 53$ & $\mathrm{E} 52 \bullet \mathrm{R} 55$ & Active \\
\hline (2) E34R & $\# 1$ & E34R $\otimes R 55$ & $\mathrm{E} 46 \bullet \mathrm{R} 53$ & $\mathrm{E} 52 \bullet \mathrm{R} 55$ & Active \\
\hline (3) E46R & $\# 2$ & $\mathrm{E} 34 \bullet \mathrm{R} 55$ & $\mathbf{E 4 6 R} \otimes R 53$ & $\mathrm{E} 52 \bullet \mathrm{R} 55$ & Active \\
\hline (4) R53E & $\# 2$ & E34・R55 & E46 @ R53E & $\mathrm{E} 52 \bullet \mathrm{R} 55$ & Active \\
\hline (5) E34R/E46R & $\# 1+2$ & $\mathbf{E 3 4 R} \otimes R 55$ & $\mathbf{E 4 6 R} \otimes R 53$ & $\mathrm{E} 52 \bullet \mathrm{R} 55$ & Active \\
\hline (6) E52R & $\# 3$ & E34・R55 & $\mathrm{E} 46 \bullet \mathrm{R} 53$ & E52R $\otimes R 55$ & Active \\
\hline (7) E34R/E52R & $\# 3+1$ & E34R $\otimes R 55$ & $\mathrm{E} 46 \bullet \mathrm{R} 53$ & E52R $\otimes R 55$ & Reduced \\
\hline (8) $\mathrm{R} 55 \mathrm{E}$ & $\# 3+1$ & $\mathrm{E} 34 \otimes \mathrm{R} 55 \mathrm{E}$ & $\mathrm{E} 46 \bullet \mathrm{R} 53$ & E52 $\otimes \mathbf{R 5 5 E}$ & Active \\
\hline (9) E46R/E52R & $\# 3+2$ & $\mathrm{E} 34 \bullet \mathrm{R} 55$ & $\mathbf{E 4 6 R} \otimes R 53$ & E52R $\otimes R 55$ & Active \\
\hline (10) R53E/E52R & $\# 3+2$ & $\mathrm{E} 34 \bullet \mathrm{R} 55$ & $\mathrm{E} 46 \otimes \mathbf{R 5 3 E}$ & E52R $\otimes R 55$ & Active \\
\hline (11) E34R/E46R/E52R & $\# 3+1+2$ & E34R $\otimes R 55$ & $\mathbf{E} 46 \otimes R 53$ & E52R $\otimes R 55$ & Inactive \\
\hline (12) R55E/R53E & $\# 3+1+2$ & E34 $\otimes \mathbf{R 5 5 E}$ & E46 @ R53E & E52 $\otimes \mathbf{R 5 5 E}$ & Reduced \\
\hline
\end{tabular}

A

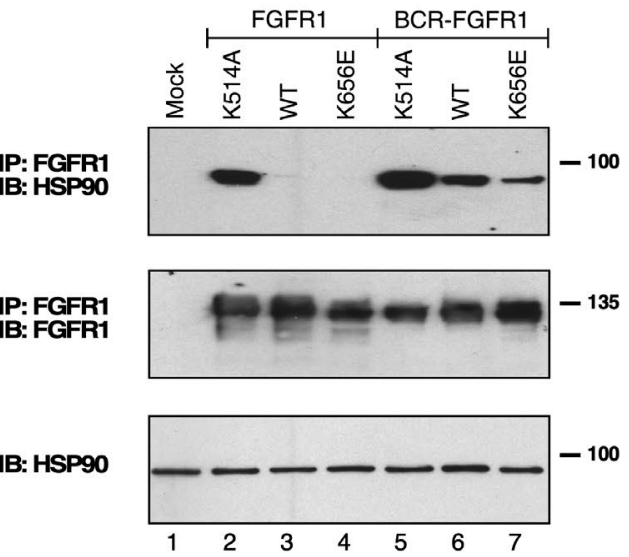

B
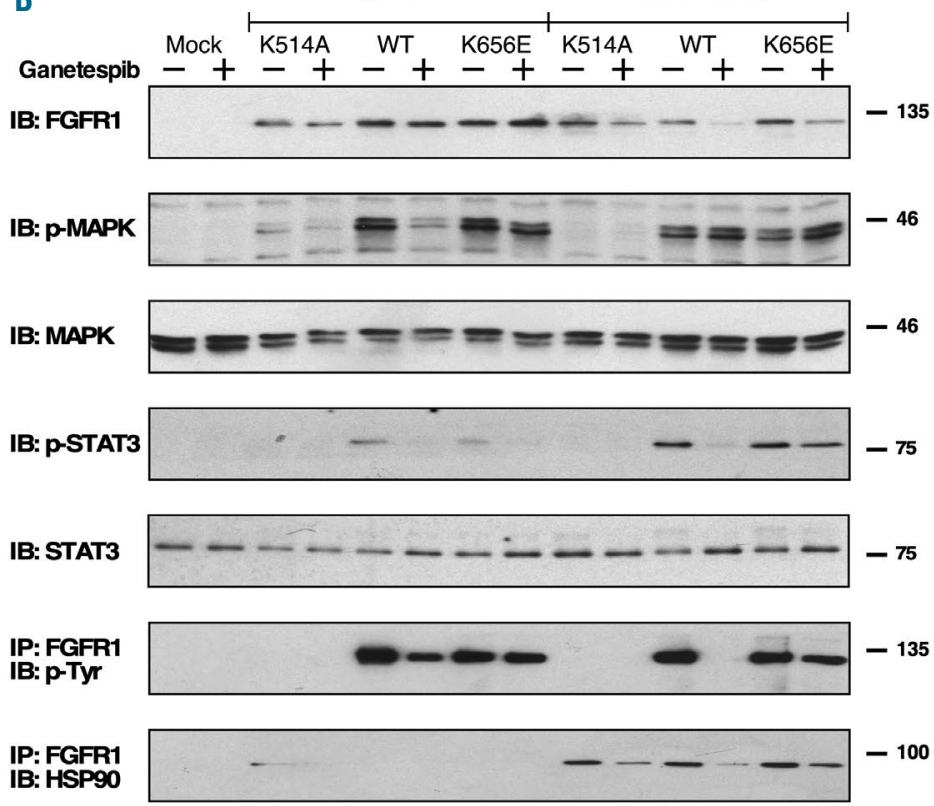

IB: GAPDH

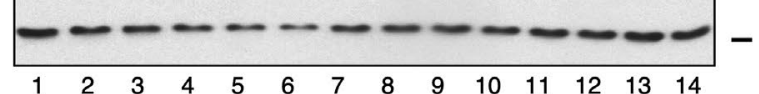

Figure 6. BCR-FGFR1 activity relies upon Hsp90. (A) Lysates from HEK293T cells expressing FGFR1 and BCR-FGFR1 derivatives were immunoprecipitated with FGFR1 and immunoblotted with anti-Hsp90 antibody (top panel). The membrane was reprobed for total FGFR1 (panel 2). The bottom panel shows the Hsp90 expression in lysate samples. (B) HEK293T cells expressing FGFR1 and BCR-FGFR1 derivatives were treated with $-/+200$ nM Ganetespib for 4 hours (h) prior to lysing. Lysates were examined for expression of the clones with anti-FGFR1 (panel 1), examined for activation of MAPK and STAT3 pathways using phospho-specific antibodies; phospho-MAPK (T202/Y204) (panel 2) and phospho-STAT3 (Y705) (panel 4). Membranes were stripped and reprobed for total MAPK and STAT3 shown in panel 3 and 5, respectively. Tyrosine phosphorylation of FGFR1 and BCR-FGFR1 derivatives was examined by immunoprecipitating FGFR1 followed by immunoblotting with anti-phosphotyrosine antibody (panel 6). The Hsp90 interaction was examined by immunoblotting with anti-Hsp90 antibody on FGFR1 immunoprecipitations (panel 7 ). The bottom control panel shows expression of GAPDH. 
matic decrease in BCR-FGFR1 expression with the addition of Ganetespib suggests that BCR-FGFR1 may be a client protein of Hsp90 and potentially uses the Hsp90 complex for protein stability within the cell.

To investigate if BCR-FGFR1 relies on Hsp90 for cell transformation, NIH3T3 cells expressing FGFR1 or BCR-
FGFR1 derivatives were treated with increasing concentrations of Ganetespib. Ganetespib concentrations were titrated to allow for a dose that effectively treated BCRFGFR1 expressing cells without harming non-expressing NIH3T3 cells. A reduction of cell transformation and focus formation is observed with increasing concentrations of
A

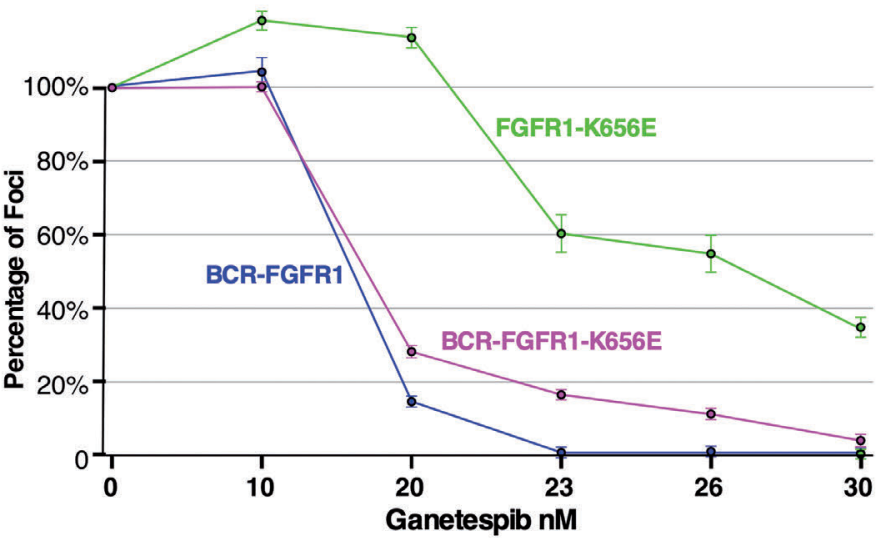

B

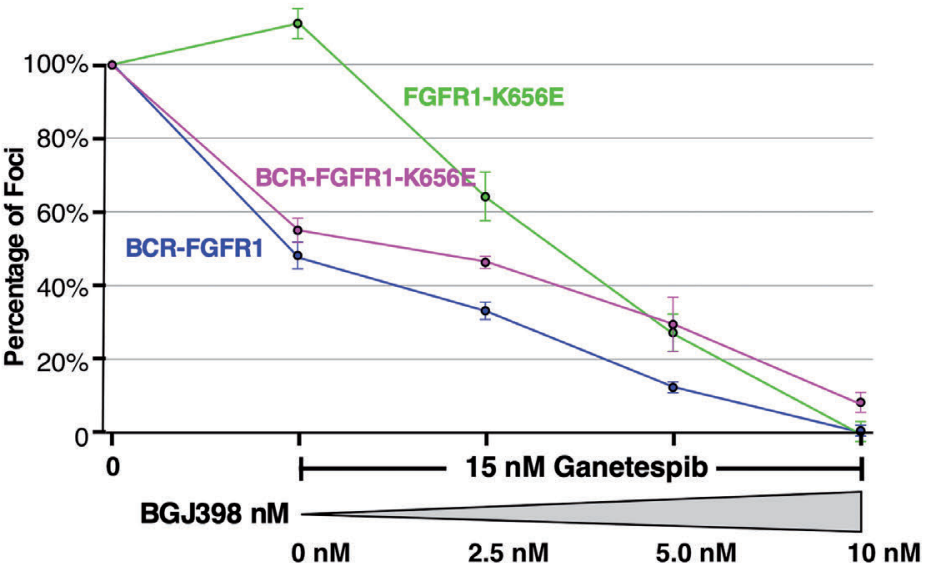

C

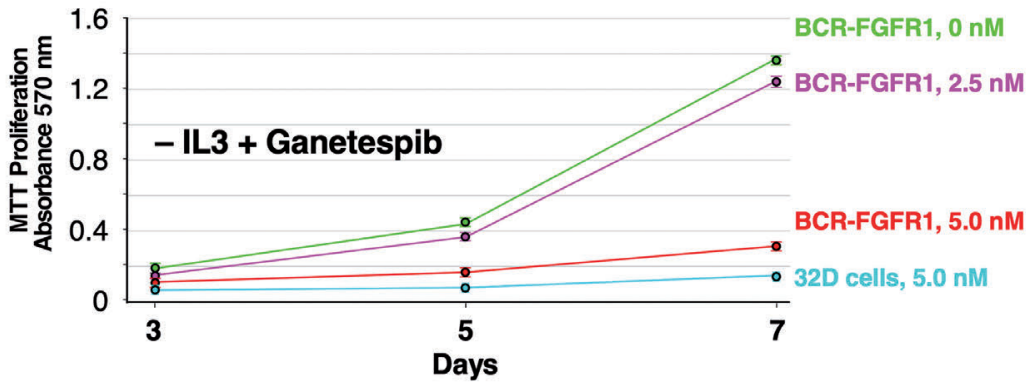

D

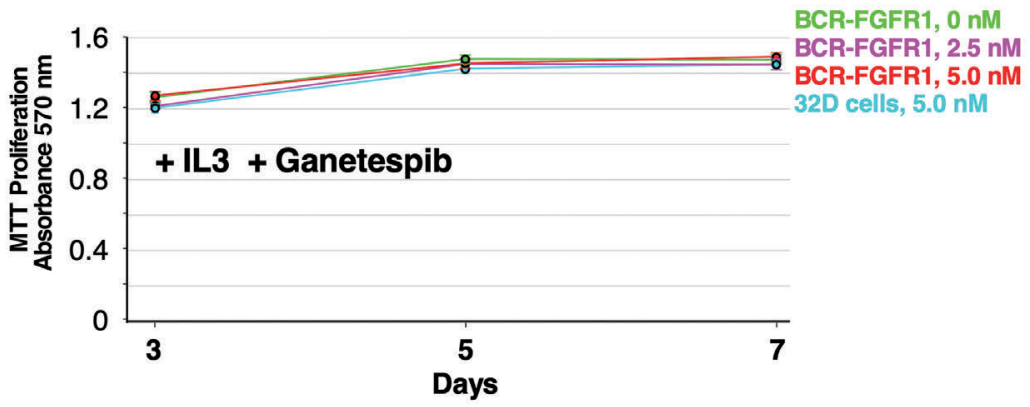

Figure 7. Effects of Ganetespib on BCR-FGFR1 activity in cellular assays. (A) NIH3T3 transformation assay. Cells expressing FGFR1-K656E, BCR-FGFR1 and BCRFGFR1-K656E were treated with increasing concentrations of the Hsp90 inhibitor, Ganetespib (10, 20, 23, 26 and $30 \mathrm{nM}$ ). After 14 days (d), foci were scored and each sample was normalized by transfection efficiency set to $100 \%$ as the starting value under conditions of no Gantespib. Mock transfected cells and cells expressing FGFR1 were also included in this assay; no foci were observed. (B) Effects of Ganetespib (15 nM) together with increasing concentrations of FGFR-specific TKI BGJ398 in NIH3T3 transformation assays. Quantitation as in (A) above. Mock transfected cells and cells expressing FGFR1 were also included in this assay; again, no foci were observed (C and D). Ganetespib inhibits 32D cell proliferation stimulated by BCR-FGFR1. $32 \mathrm{D}$ control cells and cells selectively expressing BCRFGFR1 were cultured in panel $\mathrm{C}$ in the absence of IL-3 and, in panel $\mathrm{D}$, in the presence of IL-3, together with Ganetespib at 0, 2.5, and $5.0 \mathrm{nM}$. Cells expressing FGFR1 were also included in this assay and showed no cell proliferation in the absence of IL-3, as shown earlier (Figure $3 \mathrm{~A}$ and $\mathrm{D}$ ). Cell viability was determined by MTT metabolic assay. All control cells and cell lines were assayed in triplicate. Standard error is shown. 
Ganetespib (Figure 7A). The BCR-FGFR1 derivatives were more sensitive to the Ganetespib than FGFR1-K656E, possibly due to the lack of HSP90 association with FGFR1K656E (Figure 6).

Furthermore, in order to determine if the effects of Ganetespib were synergistic with FGFR inhibition, a combinatorial experiment was performed on NIH3T3 cells expressing either BCR-FGFR1 or FGFR1 derivatives, which were simultaneously treated with Ganetespib and a TKI. These cells were treated with a constant $15 \mathrm{nM}$ Ganetespib and were dosed with increasing concentrations of BGJ398, a potent FGFR inhibitor (Figure 7B). A potentially synergistic effect is observed between Ganetespib and BGJ398, as foci production among cells expressing either BCR-FGFR1 or FGFR1 derivatives dramatically decreases. These data suggest that the use of Hsp90 inhibition combined with TKI treatment may be beneficial for BCR-FGFR1 expressing cells.

The effect of Ganetespib was also examined using 32D IL-3-dependent cells. The IL-3-independent proliferation of the BCR-FGFR1 expressing 32D cells was reduced by treatment with Ganetespib as measured by MTT assay (Figure 7C). The Ganetespib did not affect the normal dependence of the cells on IL-3, as shown in Figure 7D. Therefore, these data suggest that BCR-FGFR1 is dependent on the molecular chaperone Hsp90 for cellular transformation.

\section{Discussion}

Considerable advances have been made in our understanding of the molecular basis of hematological cancers. Since the identification of BCR-ABL almost 60 years ago, ${ }^{29}$ over 500 oncogenic translocations have been identified in hematopoietic cancers alone, which emphasizes the importance of identifying and characterizing these oncogenic drivers. ${ }^{30}$ With the emergence of personalized medicine, the characterization of activators of SCLL, such as BCR-FGFR1, is critical in determining additional therapeutic targets. Although the use of TKI to treat SCLL is becoming more commonplace, TKI treatment often results in drug resistance in patients, highlighting the need for additional therapies for SCLL. ${ }^{31}$

\section{Biological and biochemical characterization of BCR-FGFR1}

Through the data presented, we were able to extensively characterize the fusion protein BCR-FGFR1. We demonstrate that the $\mathrm{N}$-terminal fusion of $\mathrm{BCR}$ results in constitutive activation of FGFR1. Through our cell signaling studies, we demonstrate that BCR-FGFR1 activates ERK/MAPK and JAK/STAT pathways, and possesses transforming activity in NIH3T3 cells (Figure 1-2). However, BCR-FGFR1-K514A was unable to activate either pathway, or transform NIH3T3 cells, indicating that BCR-FGFR1 relies on the kinase activity of FGFR1 for activation. Furthermore, 32D cells expressing BCR-FGFR1 or BCR-FGFR1-K656E were able to proliferate in the absence of IL-3, indicating the oncogenic potential of this fusion protein (Figure 3).

LC-MS/MS data additionally demonstrate that BCRFGFR1 relies on the kinase activity of FGFR1 for oncogenic activity. Phosphorylation on key tyrosine residues in the FGFR1 kinase domain, including Y653 and Y654 in the activation loop, is observed in BCR-FGFR1 and BCRFGFR1-K656E, which is absent in kinase-dead BCRFGFR1-K514A (Figure 4). Although novel phosphorylation sites were detected on BCR, these phosphoacceptor sites do not appear to be critical for the cell transformation or oncogenic ability of BCR-FGFR1 (Table 1).

\section{Novel therapeutic targets for SCLL induced by BCR-FGFR1}

Currently, hematopoietic stem cell transplantation is the primary curative option for patients who have SCLL. Here, we have described two novel potential therapeutic approaches: disruption of ionic bonding that stabilizes BCR oligomerization, and inhibition of the chaperonin Hsp90 complex.

The coiled-coil oligomerization domain of BCR has previously been demonstrated to be essential for the transforming ability of BCR-ABL. ${ }^{21}$ However, the requirement of electrostatic interactions within the coiled-coil domain for oligomerization has not been investigated. Here, we have described a novel inhibition of BCR-FGFR1 mediated cell transformation through abolishing three salt bridge interactions in the coiled-coil domain of BCR. This ablation of cell transformation is seen through focus forming assays, as the disruption of these salt bridges in the BCR-FGFR1 mutant E34R/E46R/E52R (mutant 11) almost completely abolishes focus formation (Figure 5). Furthermore, the intrahelical Salt Bridge \#3, formed by residue Glu52 with Arg55 in BCR, plays a crucial role in providing stability for the coiled-coil domain in BCR. The apparent ability of Arg55 to form complex salt bridges with Glu34 and Glu52 highlights the potential importance of cooperativity exhibited by complex salt bridges and their contribution to protein stability. ${ }^{32}$ Taken together, these data suggest that these three salt bridges provide a critical role in the activation of BCR-FGFR1. The potential loss of oligomerization and near absence in focus formation suggests that the coiled-coil domain of BCR is an attractive therapeutic target for SCLL.

Additionally, we have shown that BCR-FGFR1 is a client of the Hsp90 chaperone complex, and potentially uses this complex to avoid proteasomal degradation in the cell. Previous work has shown that the FGFR1OP2-FGFR1 fusion is also a client of the Hsp90 complex, and that targeting the Hsp90 complex resulted in reduced activity of this fusion protein. ${ }^{33}$ The interaction and dependence of BCR-FGFR1 on the Hsp90 complex is established through cell transformation assays, and analysis of downstream cell signaling (Figure 6-7). A decrease in overall expression of BCR-FGFR1 is detected with the addition of Ganetespib, a potent Hsp90 inhibitor. Furthermore, BCRFGFR1 expressing cells treated with Ganetespib displayed a reduction in STAT and MAPK activation, and no FGFR1 phosphorylation. Additionally, the transformation ability of cells expressing BCR-FGFR1 decreases when treated with increasing concentrations of Ganetespib (Figure 7A), indicating that this fusion protein relies on the Hsp90 complex for cellular stability, and is sensitive to Hsp90 inhibition. We also examined the combined effects of Ganetespib with the FGFR inhibitor BGJ398 in NIH3T3 cell transformation assays (Figure 7B), and in 32D cell proliferation assays in the absence and presence of IL-3 (Figure 7C-D). Taken together, these data show that BCRFGFR1 may rely on the Hsp90 molecular chaperone complex to avoid proteasomal degradation. ${ }^{34}$ 
Through this work, we have described potential approaches to inhibit BCR-FGFR1 induced SCLL. Although TKI therapies have been used to traditionally treat certain hematopoietic cancers, their use has often resulted in drug resistance. Recently it has been discovered that FGFR1 fusion kinases are associated with the upregulation of MYC, which drives SCLL. ${ }^{35}$ Targeting the MYC complex in addition to chemotherapy, and the approaches described here may also be therapeutically beneficial for patients with SCLL.

\section{Relevance for $B C R-A B L$ driven cancers}

Since the initial characterization of $B C R-A B L$, the emergence of cancer genome sequencing has played a vital role in the detection of other translocation-induced malignances. $B C R-A B L$ is detected in $95 \%$ of CML cases, and variants of this gene exist with alternative breakpoints. The most commonly found transcript is the $B C R-A B L$ p210 variant which contains a breakpoint of BCR exon 13 fused to exon 1 of ABL. ${ }^{10}$ In comparison to BCR-FGFR1, $B C R-A B L$ retains more of the BCR gene in this fusion. However, both fusions retain the oligomerization domain, putative serine threonine/kinase domain and GEF domain. Due to the similarity between these fusions, we propose that the work described here will be relevant to $B C R-A B L$ fusions as well. In particular, the inhibition of the BCR coiled-coil domain through salt bridge disruption may be an additional therapeutic target for BCR-ABL. Furthermore, retention of the GEF domain in BCR-FGFR1 has been shown to result in reduced leukemogenesis. ${ }^{36}$

Although TKI are usually the first line of treatment for CML, many patients will require concurrent forms of ther- apy to ensure complete remission. ${ }^{37} \mathrm{Hsp} 90$ inhibition has become an attractive therapeutic target in treating $B C R-A B L$ induced CML. More recently, aminoxyrone was shown to be effective in inhibiting imatinib resistant CML. ${ }^{37}$ While Ganetespib binds to the N-terminal ATP binding site in Hsp90, Aminoxyrone binds to the C-terminal tail of Hsp90, preventing its dimerization. The combination of Hsp90 inhibition with traditional chemotherapy and TKI treatment may be beneficial in patients with CML or SCLL. These recent findings again highlight the importance of the characterization of oncogenic translocations as well as the development of additional therapeutic targets to treat therapy-refractory leukemia.

\section{Acknowledgements}

We thank all current lab members particularly Juyeon Ko, Fangda Li, and Clark Wang for advice and encouragement, Leo Gallo and Dan Crocker for additional support, and Alexandre R. Campos from Sanford Burnham Prebys (SBP) Medical Discovery Institute for analysis of samples by mass spectrometry. MNP gratefully acknowledges support from a UC San Diego San Diego Fellowship, and DJD gratefully acknowledges generous philanthropic support from the UC San Diego Foundation. Support to the SBP Proteomics Facility from grant P3O CA030199 from the National Institutes of Health is also gratefully acknowledged.

\section{Dedication}

MNP dedicates this work in memory of her parents Swarnakanthie Peiris (1947-2005) and Donald Peiris (19492018), as this work would not have been possible without their inspiration, love and support.

\section{References}

1. Gallo LH, Nelson KN, Meyer AN, Donoghue DJ. Functions of Fibroblast Growth Factor Receptors in cancer defined by novel translocations and mutations. Cytokine Growth Factor Rev. 2015;26(4):425-449.

2. Macdonald D, Reiter A, Cross NC. The 8p11 myeloproliferative syndrome: a distinct clinical entity caused by constitutive activation of FGFR1. Acta Haematol. 2002;107(2):101-107.

3. Nelson KN, Peiris MN, Meyer AN, et al. Receptor tyrosine kinases: translocation partners in hematopoietic disorders. Trends Mol Med. 2017;23(1):59-79.

4. Khodadoust MS, Luo B, Medeiros BC, et al. Clinical activity of ponatinib in a patient with FGFR1-rearranged mixed-phenotype acute leukemia. Leukemia. 2016;30(4):947950.

5. Verstovsek S, Rambaldi A, Asatiani E, et al. Phase 2, open-label, multicenter study to evaluate the efficacy and safety of INCB054828 in patients with myeloid/lymphoid neoplasms with fibroblast growth factor receptor 1 (FGFR1) rearrangement. Cancer Res. 2017;77(13 Suppl):Abstract nr CT057.

6. Cuesta-Dominguez A, Ortega M, Ormazabal C, et al. Transforming and tumorigenic activity of JAK2 by fusion to BCR: molecular mechanisms of action of a novel BCR-JAK2 tyrosine-kinase. PLoS One. 2012;7(2):e32451.
7. Ballerini P, Struski S, Cresson C, et al. RET fusion genes are associated with chronic myelomonocytic leukemia and enhance monocytic differentiation. Leukemia. 2012;26(11):2384-2389.

8. Baxter EJ, Hochhaus A, Bolufer P, et al. The $\mathrm{t}(4 ; 22)(\mathrm{q} 12 ; \mathrm{q} 11)$ in atypical chronic myeloid leukaemia fuses BCR to PDGFRA. Hum Mol Genet. 2002;11(12):1391-1397.

9. Peiris MN, Li F, Donoghue DJ. BCR: a promiscuous fusion partner in hematopoietic disorders. Oncotarget. 2019;10(28):27382754

10. Laurent E, Talpaz M, Kantariian H, Kurzrock $\mathrm{R}$. The BCR gene and philadelphia chromosome-positive leukemogenesis. Cancer Res. 2001;61(6):2343-2355.

11. Hart KC, Robertson SC, Kanemitsu MY, et al. Transformation and Stat activation by derivatives of FGFR1, FGFR3, and FGFR4. Oncogene. 2000;19(29):3309-3320.

12. Hu Y, Fang X, Dunham SM, et al. $90-\mathrm{kDa}$ ribosomal S6 kinase is a direct target for the nuclear fibroblast growth factor receptor 1 (FGFR1): role in FGFR1 signaling. J Biol Chem. 2004:279(28):29325-29335.

13. Gallo LH, Meyer AN, Motamedchaboki K, et al. Novel Lys63-linked ubiquitination of IKKbeta induces STAT3 signaling. Cell Cycle. 2014;13(24):3964-3976.

14. Meyer AN, McAndrew CW, Donoghue DJ. Nordihydroguaiaretic acid inhibits an activated fibroblast growth factor receptor 3 mutant and blocks downstream signaling in multiple myeloma cells. Cancer Res. 2008;68(18):7362-7370.
15. Nelson KN, Meyer AN, Wang CG Donoghue DJ. Oncogenic driver FGFR3TACC3 is dependent on membrane trafficking and ERK signaling. Oncotarget. 2018;9(76):34306-34319.

16. Nelson KN, Meyer AN, Siari A, et al Oncogenic gene fusion FGFR3-TACC3 is regulated by tyrosine phosphorylation. Mol Cancer Res. 2016;14(5):458-469.

17. Mulcahy LS, Smith MR, Stacey DW. Requirement for ras proto-oncogene function during serum-stimulated growth of NIH 3T3 cells. Nature. 1985;313(5999):241243.

18. Daley GQ, Baltimore D. Transformation of an interleukin 3-dependent hematopoietic cell line by the chronic myelogenous leukemia-specific P210bcr/abl protein. Proc Natl Acad Sci U S A. 1988;85(23):9312-9316.

19. Ma G, Lu D, Wu Y, et al. Bcr phosphorylated on tyrosine 177 binds Grb2. Oncogene. 1997;14(19):2367-2372

20. Roumiantsev S, Krause DS, Neumann CA et al. Distinct stem cell myeloproliferative/ $\mathrm{T}$ lymphoma syndromes induced by ZNF198FGFR1 and BCR-FGFR1 fusion genes from 8p11 translocations. Cancer Cell. 2004;5(3):287-298.

21. McWhirter JR, Galasso DL, Wang JY. A coiled-coil oligomerization domain of $\mathrm{Bcr}$ is essential for the transforming function of $\mathrm{Bcr}-\mathrm{Abl}$ oncoproteins. Mol Cell Biol. 1993;13(12):7587-7595.

22. Cetinbas N, Huang-Hobbs H, Tognon C, et al. Mutation of the salt bridge-forming residues in the ETV6-SAM domain interface 
blocks ETV6-NTRK3-induced cellular transformation. J Biol Chem. 2013;288(39):2794027950.

23. Taylor CM, Keating AE. Orientation and oligomerization specificity of the Bcr coiledcoil oligomerization domain. Biochemistry. 2005;44(49):16246-16256.

24. Zhao X, Ghaffari S, Lodish H, et al. Structure of the Bcr-Abl oncoprotein oligomerization domain. Nat Struct Biol. 2002;9(2):117-120.

25. Pettersen EF, Goddard TD, Huang CC, et al. UCSF Chimera--a visualization system for exploratory research and analysis. J Comput Chem. 2004;25(13):1605-1612

26. Taipale M, Jarosz DF, Lindquist S. HSP90 at the hub of protein homeostasis: emerging mechanistic insights. Nat Rev Mol Cell Biol. 2010;11(7):515-528.

27. Whitesell L, Lindquist SL. HSP90 and the chaperoning of cancer. Nat Rev Cancer. 2005;5(10):761-772.
28. Neckers L. Hsp90 inhibitors as novel cancer chemotherapeutic agents. Trends Mol Med. 2002;8(4 Suppl):S55-61.

29. Nowell PC, Hungerford DA. Chromosome studies on normal and leukemic human leukocytes. J Natl Cancer Inst. 1960;25:85109.

30. Nambiar M, Kari V, Raghavan SC. Chromosomal translocations in cancer. Biochim Biophys Acta. 2008;1786(2):139152.

31. Musumeci F, Greco C, Grossi G, et al Recent studies on ponatinib in cancers other than chronic myeloid leukemia. Cancers (Basel). 2018;10(11).

32. Gvritishvili AG, Gribenko AV, Makhatadze GI. Cooperativity of complex salt bridges. Protein Sci. 2008:17(7):1285-1290.

33. Jin Y, Zhen Y, Haugsten EM, Wiedlocha A. The driver of malignancy in KG-1a leukemic cells, FGFR1OP2-FGFR1, encodes an HSP90 addicted oncoprotein. Cell Signal. 2011 23(11):1758-1766

34. Gallo LH, Ko J, Donoghue DJ. The importance of regulatory ubiquitination in cancer and metastasis. Cell Cycle. 2017;16(7):634648.

35. Hu T, Wu Q, Chong Y, et al. FGFR1 fusion kinase regulation of MYC expression drives development of stem cell leukemia/lymphoma syndrome. Leukemia. 2018;32(11): 2363-2373.

36. Hu T, Chong Y, Lu S, et al. Loss of the BCRFGFR1 GEF Domain Suppresses RHOA Activation and Enhances BLymphomagenesis in Mice. Cancer Res. 2019;79(1):114-124.

37. Bhatia S, Diedrich D, Frieg B, et al. Targeting HSP90 dimerization via the $C$ terminus is effective in imatinib-resistant CML and lacks the heat shock response. Blood. 2018; 132(3):307-320 